\title{
Correcting C-Band Radar Reflectivity and Differential Reflectivity Data for Rain Attenuation: A Self-Consistent Method With Constraints
}

\author{
V. N. Bringi, T. D. Keenan, and V. Chandrasekar, Member, IEEE
}

\begin{abstract}
Quantitative use of C-band radar measurements of reflectivity $\left(Z_{h}\right)$ and differential reflectivity $\left(Z_{\mathrm{dr}}\right)$ demands the use of accurate attenuation-correction procedures, especially in convective rain events. With the availability of differential phase measurements $\left(\Phi_{d p}\right)$ with a dual-polarized radar, it is now possible to improve and stabilize attenuation-correction schemes over earlier schemes which did not use $\Phi_{d p}$. The recent introduction of constraint-based correction schemes using $\Phi_{d p}$ constitute an important advance [8], [9]. In this paper, a self-consistent, constraint-based algorithm is proposed and evaluated which extends the previous approaches in several important respects. Radar data collected by the C-POL radar during the South China Sea Monsoon Experiment (SCSMEX) are used to illustrate the correction scheme. The corrected radar data are then compared against disdrometer-based scattering simulations, the disdrometer data being acquired during SCSMEX. A new algorithm is used to retrieve the median volume diameter from the corrected $Z_{h}$, corrected $Z_{\mathrm{dr}}$, and $K_{\mathrm{dp}}$ radar measurements which is relatively immune to the precise drop axis ratio versus drop diameter relation. Histograms of the radar-retrieved $D_{o}$ compared against $D_{o}$ from disdrometer data are in remarkable good agreement lending further validity to the proposed attenuation-correction scheme, as well as to confidence in the use of $\mathrm{C}$-band radar for the remote measurement of rain microphysics.
\end{abstract}

Index Terms-Attenuation, dual-polarized, radar, rain.

\section{INTRODUCTION}

$\mathbf{I}$ T IS well known that convective storms cause significant attenuation and differential attenuation (between horizontal and vertical polarized waves) at C-band (frequency near 5.5 $\mathrm{GHz}$ ) and higher frequencies. As a consequence, radar measurements of reflectivity $\left(Z_{h}\right.$; at horizontal polarization) and differential reflectivity $\left(Z_{\mathrm{dr}}\right)$ must be corrected for rain attenuation before they can be used quantitatively (e.g., in rainfall algorithms or for hydrometeor identification).

The early approaches to attenuation-correction were iterative, correcting the $\left(Z_{h}\right.$ or $\left.Z_{\mathrm{dr}}\right)$ dates starting from the first range resolution volume (where attenuation is negligible) and proceeding to successive resolution volumes along the beam as it intercepts the rain cell and beyond [1]-[4]. Such methods are known to be

Manuscript received October 20; 2000; revised April 20, 2001. The work of V. N. Bringi and V. Chandrasekar was supported by the NASA/TRMM Grant NAG5-7717 and -7876.

V. N. Bringi and V. Chandrasekar are with the Department of Electrical and Computer Engineering, Colorado State University, Fort Collins, CO 80523-1373.

T. D. Keenan is with the Bureau of Meteorology Research Center (BMRC), Melbourne, Australia.

Publisher Item Identifier S 0196-2892(01)08119-0. unstable if the path-integrated attenuation is large and they assume that the radar is absolutely calibrated with high accuracy.

Attenuation correction procedures are greatly improved if the total path-integrated attenuation is available as a constraint, e.g., using a dual-frequency radar where one frequency is nonattenuating, while the second is attenuating [5]. Dual-polarized radars offer a total differential propagation phase $\left(\Phi_{\mathrm{dp}}\right.$; between horizontal and vertical polarizations) constraint, which is equivalent to the total path-integrated attenuation constraint because a linear relation exists between the two at typical radar frequencies $(3-10 \mathrm{GHz})$ [6]. Similarly, the total differential attenuation along the path can be constrained using $\Phi_{\mathrm{dp}}$ since a near linear relationship exists between the two [6], [7]. The correction of measured reflectivity $\left(Z_{h}\right)$ using the total $\Phi_{\mathrm{dp}}$ constraint (termed the ZPHI algorithm) was recently proposed and evaluated [8]. The correction of measured $Z_{\mathrm{dr}}$, again using the total $\Phi_{\mathrm{dp}}$ constraint was described in [9] and applied to S-band (frequency near $3 \mathrm{GHz}$ ) radar data. In this paper, a self-consistent scheme is introduced which extends the methodologies presented in [8], [9] in several important respects. Radar data collected by the C-POL radar operated by the Australian Bureau of Meteorology Research Center during the South China Sea Monsoon Experiment (SCSMEX) are used to illustrate the self-consistent scheme.

This paper is organized as follows. Section II gives a brief but relevant background on attenuation-correction procedures that rely on $\Phi_{\mathrm{dp}}$. Section III describes the standard ZPHI method and the self-consistent extensions proposed in this paper for correction of the measured $Z_{h}$. Section IV outlines the $Z_{\mathrm{dr}}$ correction procedure described in [9] and the related self-consistent method that is proposed in this paper. Section $\mathrm{V}$ describes the analysis of C-POL radar data (which have been corrected by the self-consistent scheme) for one convective rain event which are then compared with simulations based on measured raindrop size spectra during SCSMEX. Section VI uses the corrected radar data to illustrate the retrieval of the median volume diameter $\left(D_{o}\right)$ of the raindrop size distribution using a new algorithm, based in part on [10]. Histograms of $D_{o}$ from one convective rain event during SCSMEX are then compared against disdrometer data from that region.

\section{BACKGROUND}

The absorption and scattering of electromagnetic waves due to precipitation has been studied since the early forties nearly 
coincident with the beginning of radar. The extinction cross section of particles $\left(\sigma_{\text {ext }}\right)$ determines the power loss suffered by the incident wave due to absorption and scattering. If raindrops are assumed to be spherical (diameter $D$ ) and if $\lambda$ is the wavelength, then $\sigma_{\text {ext }}$ can be expressed in the term of the Mie scattering coefficients as

$$
\sigma_{\mathrm{ext}}=\frac{2 \pi}{k_{o}^{2}} \sum_{n=1}^{\infty}(2 n+1) \operatorname{Re}\left(a_{n}^{s}+b_{n}^{s}\right)
$$

The Mie coefficients $\left(a_{1}^{s}, b_{1}^{s}, a_{2}^{s}\right)$ are sufficient to approximate $\sigma_{\text {ext }}$ up to order $(D / \lambda)^{5}$. Thus, for frequencies from 3 to $5 \mathrm{GHz}$, $\sigma_{\text {ext }}$ may be expressed as [11]

$$
\begin{aligned}
\sigma_{\mathrm{ext}}= & \frac{2 \pi}{k_{o}^{2}}\left[3 \operatorname{Re}\left(a_{1}^{s}+b_{1}^{s}\right)+5 \operatorname{Re}\left(a_{2}^{s}\right)\right] \\
= & \frac{6 \pi}{\lambda}\left(\frac{\pi}{6} D^{3}\right) \\
& \cdot\left[i\left(\frac{\epsilon_{r}-1}{\epsilon_{r}+2}\right)\left\{1+\left(\frac{\pi D}{\lambda}\right)^{2}\left(t+u+\frac{5}{3} w\right)\right\}\right]
\end{aligned}
$$

where $\epsilon_{r}$ is the complex relative permittivity, and $t, u$, and $w$ are defined in [11, p. 143]. Note that "Re" stands for real part of a complex number and $i=\sqrt{-1}$. Fig. 1 shows $\sigma_{\text {ext }}$ (in square millimeters) versus drop diameter for wavelengths of 10 , 5 , and $3 \mathrm{~cm}$. A power law fit of the form $\sigma_{\mathrm{ext}}=C_{\lambda} D^{n}$ for $0.1 \leq D \leq 8 \mathrm{~mm}$ can be used as first approximation with $n=3.3,3.9$, and 4.1 at 10,5 , and $3 \mathrm{~cm}$ wavelengths, respectively. However, for large drops with $5 \leq D \leq 10 \mathrm{~mm}$, the corresponding $n$-values are $4.6,4.8$, and 4.9 , respectively. To simplify the discussion and to develop the form of the relation between attenuation and differential propagation phase, let $n \approx 4$. Note, however, that the temperature dependence of $\operatorname{Im}\left(\epsilon_{r}\right)$ in (3) will cause both $C_{\lambda}$ and $n$ to be temperature dependent [12].

If the raindrop size distribution is $N(D)$ (in units of $\mathrm{mm}^{-1}$ and $\mathrm{m}^{-3}$ ), then the specific attenuation is given by

$$
\begin{aligned}
A & =\left(4.343 \times 10^{3}\right) \int \sigma_{\mathrm{ext}}(D) N(D) d D ; \mathrm{dB} \mathrm{km}^{-1} \\
& \approx 4.343 \times 10^{3} C_{\lambda} \int D^{4} N(D) d D .
\end{aligned}
$$

To develop attenuation-correction procedures based on differential propagation phase $\left(\Phi_{\mathrm{dp}}\right)$ assume that raindrops of equivalent spherical diameter $D$ are actually oblate spheroidal with axis ratio $r(r=b / a ; a, b$ being the semi-major and semi-minor axes of the spheroid, respectively). The relation between $D$ and $r$ is well known for equilibrium drop shapes, but drop oscillations can perturb this relation [13], [14]. To a first approximation, let $r=1-\gamma D$ represent the axis ratio versus diameter relation with $\gamma$ being the slope (e.g., the equilibrium slope $\gamma=0.062$ for the Pruppacher-Beard linear fit [15], where $D$ is in millimeters). Following [16], at long wavelengths the specific differential phase $\left(K_{\mathrm{dp}}\right)$ can be expressed as

$$
\begin{aligned}
K_{\mathrm{dp}} & =\left(\frac{180}{\lambda}\right) 10^{-3} C W\left(1-\bar{r}_{m}\right) ;{ }^{\circ} \mathrm{km}^{-1} \\
& =\left(\frac{180}{\lambda}\right) 10^{-3} C W\left(\gamma D_{m}\right)
\end{aligned}
$$

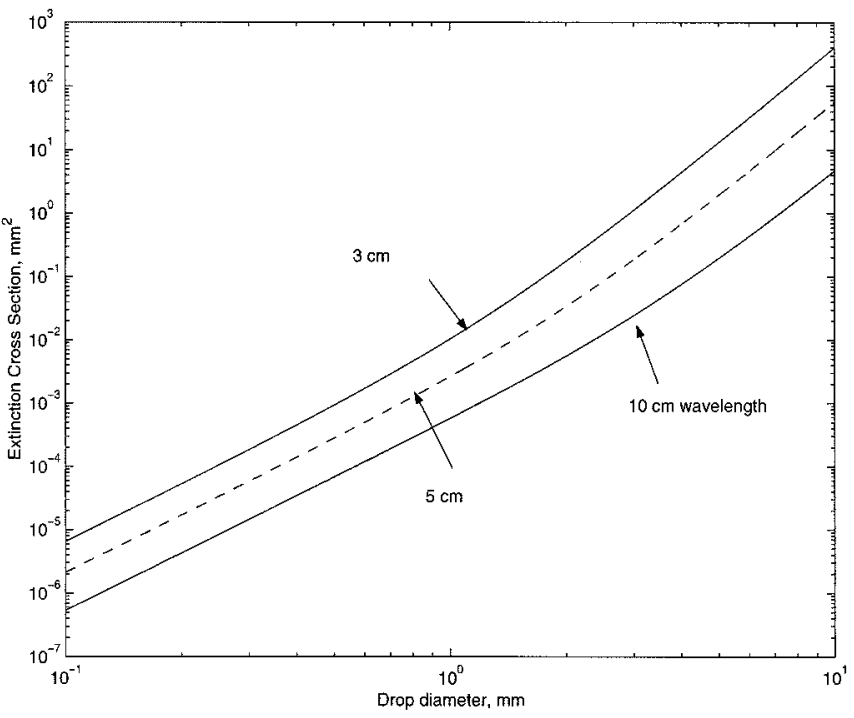

Fig. 1. Extinction cross section of spherical drops versus drop diameter using the low-frequency expansion in (3).

where $C \approx 3.75$ is both dimensionless and independent of wavelength; $W$ is the rainwater content (in $\mathrm{gm}^{-3}$ ); and $\lambda$ is in meters. The $\bar{r}_{m}$ is the mass-weighted mean axis ratio defined as

$$
\bar{r}_{m}=\frac{\int r D^{3} N(D) d D}{\int D^{3} N(D) d D}
$$

while $D_{m}$ [in millimeters, in (7)] is the mass-weighted mean diameter

$$
D_{m}=\frac{\int D^{4} N(D) d D}{\int D^{3} N(D) d D} .
$$

Since $W D_{m}$ is proportional to the fourth moment of $N(D)$, it follows from (5) that the specific attenuation $\left(A \approx A_{h}\right.$, where $A_{h}$ is the specific attenuation at horizontal polarization) is nearly linearly related to $K_{\mathrm{dp}}$ but inversely proportional to $\gamma$. In compact notation $A_{h}$ is proportional to $C_{\lambda}\left(K_{\mathrm{dp}} / \gamma\right)$ or $A_{h}=\alpha K_{\mathrm{dp}}$ where the coefficient $\alpha$ is both dependent on temperature because of $C_{\lambda}$ as well as the slope $\gamma$ of the relation between the mean axis ratio $(r)$ and equivalent diameter $(D)$. While the temperature dependence is well known [17], the sensitivity to $\gamma$ is not as well recognized. Recently, polarimetric radar algorithms for estimating $\gamma$ from measurements of $Z_{h}$, $Z_{\mathrm{dr}}$, and $K_{\mathrm{dp}}$ have been explored [10]. Attenuation-correction schemes that assume a constant value for $\alpha$ can be in error due to both temperature variations as well as variations in $\gamma$. In this paper, a self-consistent extension of the ZPHI method [8] of attenuation-correction due to rain is proposed which does not assume an a priori value for $\alpha$.

To correct the measured $Z_{\mathrm{dr}}$ for differential attenuation, the general approach is based on assuming a linear relation between the specific attenuation $\left(A_{\mathrm{dp}}=A_{h}-A_{v}\right)$ and $K_{\mathrm{dp}}$ of the form $A_{\mathrm{dp}}=\beta K_{\mathrm{dp}}$ [6], [7]. Scattering simulations based on 
gamma models for $N(D)$ show that linearity is a good approximation over a wide frequency range $(2.8-19 \mathrm{GHz})$ but that the coefficient $\beta$ is temperature-dependent at the lower frequencies varying by a factor of 2 (for $0-30{ }^{\circ} \mathrm{C}$ ) at 2.8 and $5.5 \mathrm{GHz}$ [17]. Scattering simulations also show that $\beta$ is much less sensitive to the slope $\gamma$ of the axis ratio versus $D$ relation as compared to $\alpha$ which is not unexpected since both $A_{\mathrm{dp}}$ and $K_{\mathrm{dp}}$ are differential quantities. It also follows that $A_{\mathrm{dp}}$ is expected to be linearly related to $A_{h}$, i.e., $A_{\mathrm{dp}}=(\beta / \alpha) A_{h}$. It have been suggested that $A_{\mathrm{dp}}$ be first estimated using $A_{\mathrm{dp}}=\beta K_{\mathrm{dp}}$ together with a $Z_{\mathrm{dr}}$ constraint on the far side of the rain cell, and then $A_{h}$ be derived from $A_{h}=(\alpha / \beta) A_{\mathrm{dp}}$ [9]. In this paper, a self-consistent extension of the $Z_{\mathrm{dr}}$ constraint method [9] is proposed assuming $Z_{h}$ has already been corrected.

Several articles have noted that the attenuation (and differential attenuation) due to "giant" raindrops along the propagation path result in values of $\alpha$ (and $\beta$ ) that are nearly double the theoretical values expected from scattering simulations [9], [18], [19]. A simple explanation is given here which follows from referring to Fig. 1 and noting that $\sigma_{\text {ext }}$ varies closer to $D^{5}$ for drops with diameters from 5 to $10 \mathrm{~mm}$ rather than as $D^{4}$, as assumed in (5). Further, if $N(D)$ is assumed to be an exponential distribution

$$
N(D)=N_{o} \exp \left(-3.67 D / D_{o}\right)
$$

where $D_{o}$ is the median volume diameter, then from (4), $A$ is proportional to $D_{0}^{6}$ whereas $K_{\mathrm{dp}}$ from (7) is still proportional to $W D_{m}$ or to $D_{o}^{5}$. It follows that $A$ is proportional to $D_{o} K_{\mathrm{dp}}$ and, thus, the coefficient $\alpha$ (in $A_{h}=\alpha K_{\mathrm{dp}}$ ) will increase with $D_{\circ}$. Scattering simulations show that this dependence of $\alpha$ on $D_{o}$ only occurs when $D_{o}$ exceeds $2.5 \mathrm{~mm}$. Similarly, $\beta$ will increase as $D_{0}$ increases beyond $2.5 \mathrm{~mm}$ [9], [19]. Such "giant" drops can be detected (based, in part, on scattering differential phase measurements at $\mathrm{C}$-band) and $\beta$ values can be empirically increased locally to account for the enhanced differential attenuation [19].

\section{SELF-CONSISTENT ZPHI METHOD FOR ATTENUATION CORRECTION}

The ZPHI method is based on "rain profiling" algorithms developed for space borne radar [20] and has been adapted for ground-based radar [8]. In the case of spaceborne radar, the ocean surface acts as a reference whose radar cross section is stable and known. By comparing the backscattering signal from the surface in the presence of rain relative to rain-free areas, the path attenuation can be estimated. A number of different algorithms have been analyzed in [20] with somewhat different properties. In the case of ground radars with polarimetric capability the value of $\Phi_{\mathrm{dp}}\left(r_{m}\right)$ at range locations beyond the attenuating rain cell is used as a constraint.

The ZPHI and similar rain-profiling methods are based on [21]. Before describing the self-consistent extension to the ZPHI method proposed in this paper, the standard ZPHI method [8] is first outlined. The specific attenuation $A_{h}\left(\right.$ in $\left.\mathrm{dB} \mathrm{km}^{-1}\right)$ is related to $Z_{h}$ (in $\mathrm{mm}^{6} \mathrm{~m}^{-3}$ ) by means of power law

$$
A_{h}(r)=a\left[Z_{h}(r)\right]^{b} .
$$

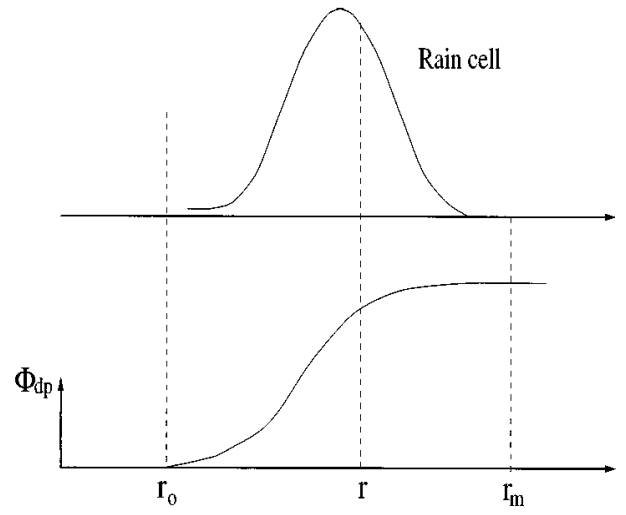

Fig. 2. Illustration related to the ZPHI method. The ranges $r_{o}, r$, and $r_{m}$ are shown relative to an idealized rain cell and the $\Phi_{\mathrm{clp}}$ range profile.

For the gamma form of $N(D)$, scattering simulations show that $b$ is very nearly constant for a given frequency (at C-band $b=$ 0.78 ), whereas $a$ depends on temperature as well as $N_{o}^{1-b}$ [8]. The ZPHI method of attenuation correction does not involve $a$, but it does assume that $b$ is constant and that $N_{o}$ is independent of $r$. Next, a linear relation between $A_{h}$ (in $\mathrm{dB} \mathrm{km}^{-1}$ ) and $K_{\mathrm{dp}}\left(\right.$ in $\left.^{\circ} \mathrm{km}^{-1}\right)$ is assumed with known $\alpha$-coefficient $\left(A_{h}(r)=\right.$ $\left.\alpha K_{\mathrm{dp}}(r)\right)$. Simulations show that linearity is an excellent assumption when $D_{\circ} \leq 2.5 \mathrm{~mm}$, the exponent varying between 0.96 and 1.02 [8], [17] at C-band. As discussed earlier, $\alpha$ depends on temperature, and on the assumed drop axis ratio versus diameter relation. A thorough simulation of the ZPHI method considering these various assumptions is given in [8].

The measured reflectivity $Z^{\prime}$ at range $r$ is expressed as (for ease of notation the subscript $h$ will be dropped for now)

$$
Z^{\prime}(r)=Z(r) e^{-0.46 A r}
$$

where $Z$ is the intrinsic reflectivity (in units of $\mathrm{mm}^{6} \mathrm{~m}^{-3}$ with $r$ in $\mathrm{km}$ ). For an inhomogeneous path, (12) can be generalized as

$$
Z^{\prime}(r)=Z(r) e^{-0.46 \int_{0}^{r} A(s) d s} .
$$

Referring to Fig. 2, the solution to $A(r)$ by the ZPHI method is given as [8] (with subscript $h$ reintroduced)

$$
A_{h}(r)=\frac{\left[Z_{h}^{\prime}(r)\right]^{b}\left[10^{0.1(b \alpha) \Delta \Phi_{\mathrm{cp}}\left(r_{o} ; r_{m}\right)}-1\right]}{I\left(r_{o} ; r_{m}\right)+\left[10^{0.1(b \alpha) \Delta \Phi_{\mathrm{clp}}\left(r_{o} ; r_{m}\right)}-1\right] I\left(r ; r_{m}\right)}
$$

where $\Delta \Phi_{\mathrm{dp}}$ is the change in differential propagation phase from $r_{o}$ to $r_{m}$. Note that the integral of $A_{h}(r)$ from $r_{o}$ to $r_{m}$ equals $(\alpha / 2) \Delta \Phi_{\mathrm{dp}}\left(r_{o} ; r_{m}\right)$. To reiterate, the exponent $b$ is defined in (11) while $\alpha$ is the coefficient in the linear relation $A_{h}=\alpha K_{\mathrm{dp}}$. The derivation of (14) is not given in [8] but the intermediate step, i.e., without the $\Phi_{\mathrm{dp}}$ constraint, can be found in [20]. The complete derivation of (14) is given in [22, Ch. 7]. The function $I\left(r ; r_{m}\right)$ is defined as

$$
I\left(r ; r_{m}\right)=0.46 b \int_{r}^{r_{m}}\left[Z_{h}^{\prime}(r)\right]^{b} d r .
$$

Equation (14) gives a solution for the specific attenuation $A_{h}$ (in $\mathrm{dB} \mathrm{km}^{-1}$ ) at each $r$ (in $\mathrm{km}$ ) from $r_{o}$ to $r_{m}$ in terms of the measured reflectivity $Z_{h}^{\prime}(r)$ (in $\mathrm{mm}^{-6} \mathrm{~m}^{-3}$ ), the measured $\Delta \Phi_{\mathrm{dp}}$ 
across the rain cell (from $r_{o}$ to $r_{m}$ in degrees), and the coefficients $\alpha$ and $b$. A detailed simulation and sensitivity was conducted by Testud et al. [8]. In particular, the retrieval of $A_{h}$ does not depend on the radar system constant, as pointed out in [8]. Once $A_{h}(r)$ is calculated at each range location from $r_{o}$ to $r_{m}$, the measured reflectivity can be corrected using

$$
10 \log _{10}\left[Z_{h}(r)\right]=10 \log _{10}\left[Z_{h}^{\prime}(r)\right]+2 \int_{r_{o}}^{r} A_{h}(s) d s
$$

yielding an attenuation-correction algorithm that is constrained by the change in differential propagation phase across the range interval $r_{o}-r_{m}$. This algorithm is sensitive to the dependence of the $\alpha$-coefficient on drop temperature and to the form of the mean axis ratio versus diameter relation.

The self-consistent extension to the ZPHI method proposed here does not assume a priori a constant value for $\alpha$. Rather, $\alpha$ is assumed to lie in a predetermined range $\left(\alpha_{\min }, \alpha_{\max }\right)$ which establishes the lower and upper bound values. These can be obtained from scattering simulations for a given range of temperature [17] and the slope $\gamma$ (of the axis ratio-diameter relation). For each $\alpha$, a "constructed" differential propagation phase, $\Phi_{\mathrm{dp}}^{c}(r ; \alpha)$, is computed as

$\Phi_{\mathrm{dp}}^{c}(r ; \alpha)=2 \int_{r_{0}}^{r} \frac{A_{h}(s ; \alpha)}{\alpha} d s ; \quad \alpha_{\min } \leq \alpha \leq \alpha_{\max }$

where $A_{h}(s ; \alpha)$ is obtained from (14) for each value of $\alpha$. The optimal $\alpha$ is selected by minimizing the difference between the constructed $\Phi_{\mathrm{dp}}^{c}$ range profiles and a filtered version $\left(\Phi_{\mathrm{dp}}^{\mathrm{filt}}\right)$ of the measured $\Phi_{\mathrm{dp}}$ over the range $\left(r_{o}, r_{m}\right)$

$$
\text { Error }=\sum_{j=1}^{N}\left|\Phi_{\mathrm{dp}}^{\mathrm{filt}}\left(r_{j}\right)-\Phi_{\mathrm{dp}}^{c}\left(r_{j} ; \alpha\right)\right|
$$

where $r_{1} \equiv r_{o}$ and $r_{N} \equiv r_{m}$. As discussed in [23], it is possible to adaptively filter the "raw" differential phase data so that backscatter phase shift can be corrected, at the same time yielding a smoothed $\Phi_{\mathrm{dp}}$ range profile that can be used in minimizing the error in (18).

To illustrate this minimization procedure, radar data from the BMRC C-POL radar operating near a frequency of $5.5 \mathrm{GHz}$ (C-band) are used [24]. Fig. 3 shows an example range profile of (a) the measured reflectivity $Z_{h}^{\prime}$, (b) the measured differential reflectivity $Z_{\mathrm{dr}}^{\prime}$, and (c) the measured $\Phi_{\mathrm{dp}}$ and its filtered version $\Phi_{\mathrm{dp}}^{\text {filt }}$. The data are from a typical convective rain cell from SCSMEX. Fig. 4(a) illustrates $\Phi_{\mathrm{dp}}^{c}(r ; \alpha)$ for two extreme values of $\alpha$ together with $\Phi_{\mathrm{dp}}^{\text {filt }}$, while Fig. 4(b) shows the error versus $\alpha$ indicating that an optimal value for $\alpha\left(\alpha_{\mathrm{opt}}\right)$ was found. Fig. 4(c) shows the optimal constructed profile $\Phi_{\mathrm{dp}}^{c}\left(r ; \alpha_{\mathrm{opt}}\right)$, compared with the "raw" measured $\Phi_{\mathrm{dp}}$, and excellent agreement may be noted. The range profile of the retrieved $A_{h}(r)$ using (14) with $\alpha_{\text {opt }}$ (note $b \approx 0.8$ at C-band) is shown in Fig. $4(\mathrm{~d})$, together with the $K_{\mathrm{dp}}$ profile, which is one-half the range derivative of $\Phi_{\mathrm{dp}}^{\text {filt }}$ [see Fig. 3(c)]. The retrieved $A_{h}(r)$ is then used in (16) to arrive at the corrected $Z_{h}(r)$ shown in Fig. 3(a).

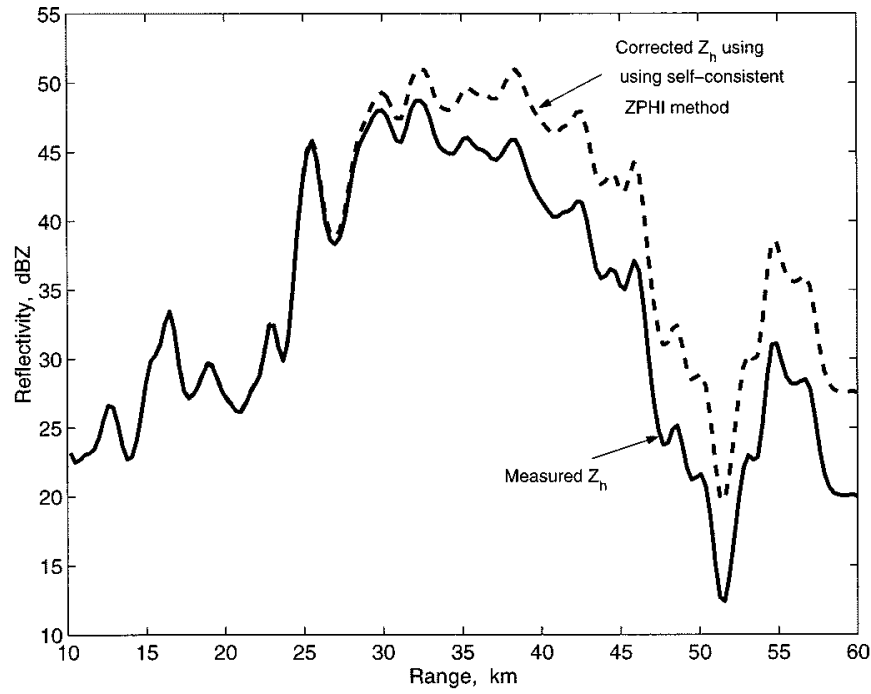

(a)

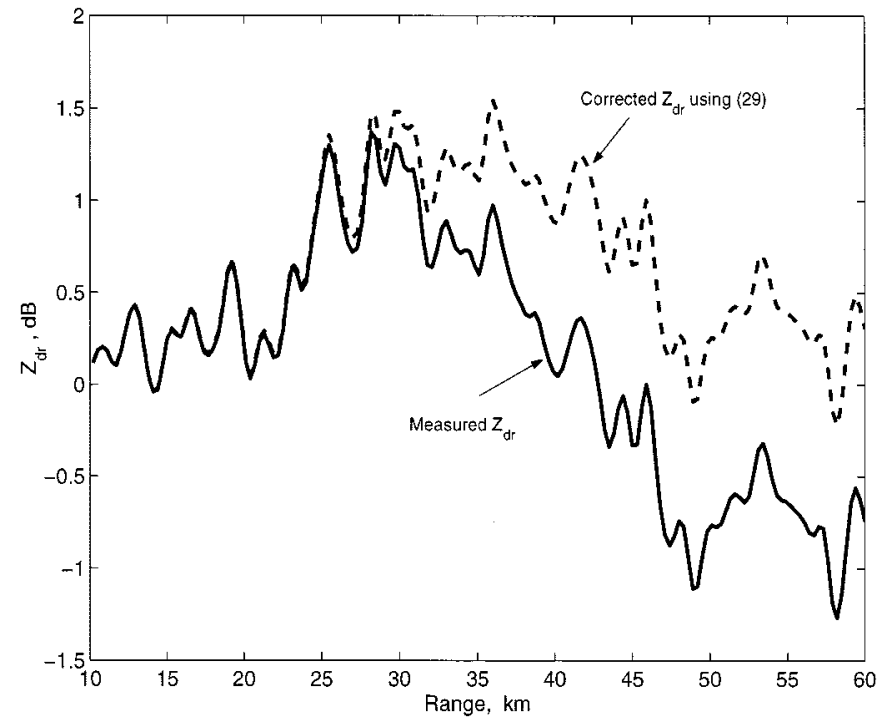

(b)

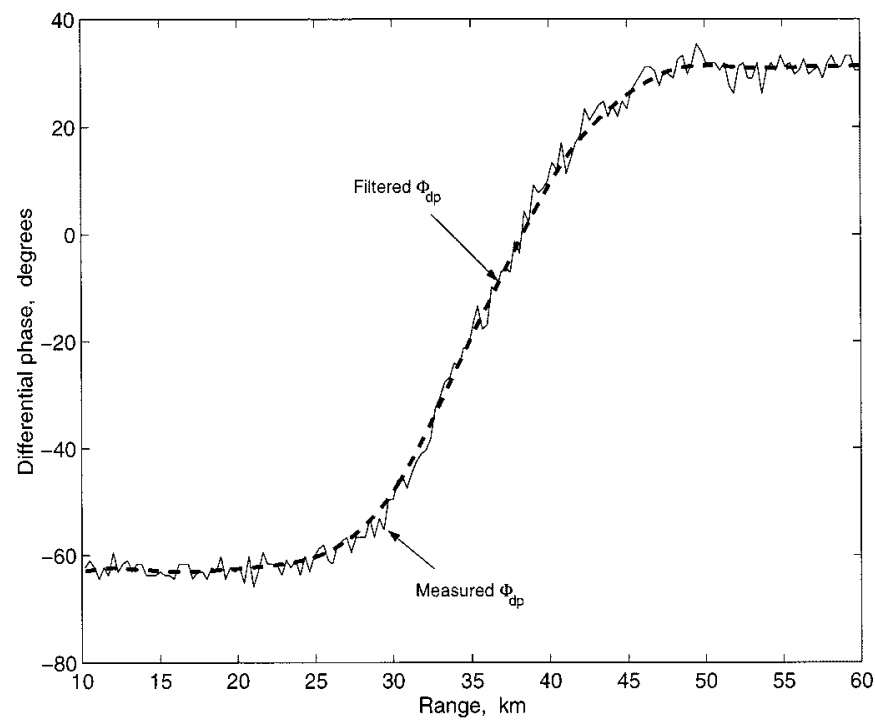

(c)

Fig. 3 (a) Measured and corrected $Z_{h}$ versus range. (b) Measured $Z_{\mathrm{dr}}$ and corrected $Z_{\mathrm{clr}}$ versus range. (c) Measured $\Phi_{\mathrm{clp}}$ and the filtered $\Phi_{\mathrm{clp}}$. 


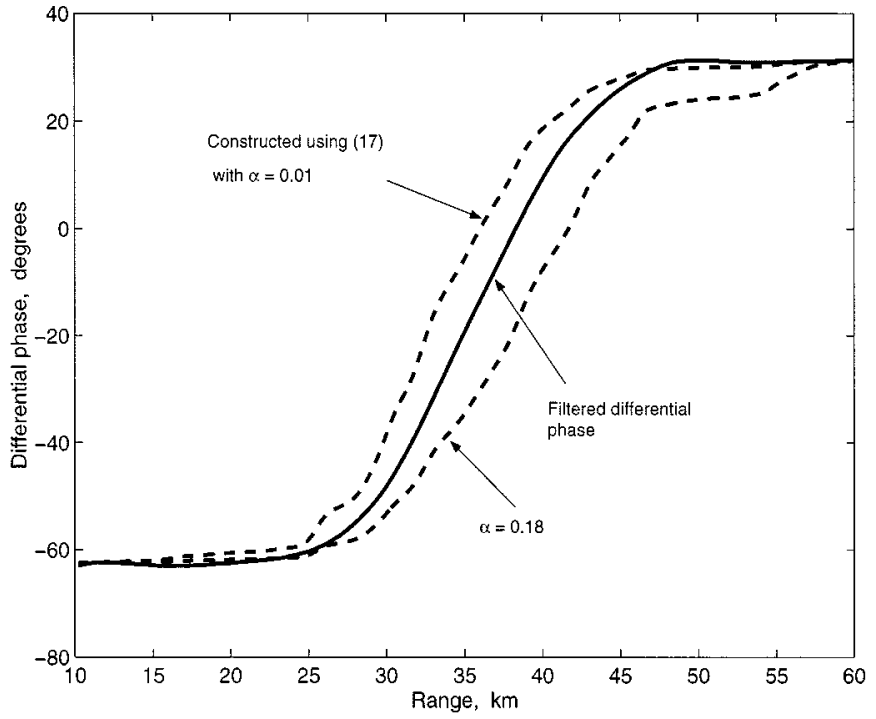

(a)

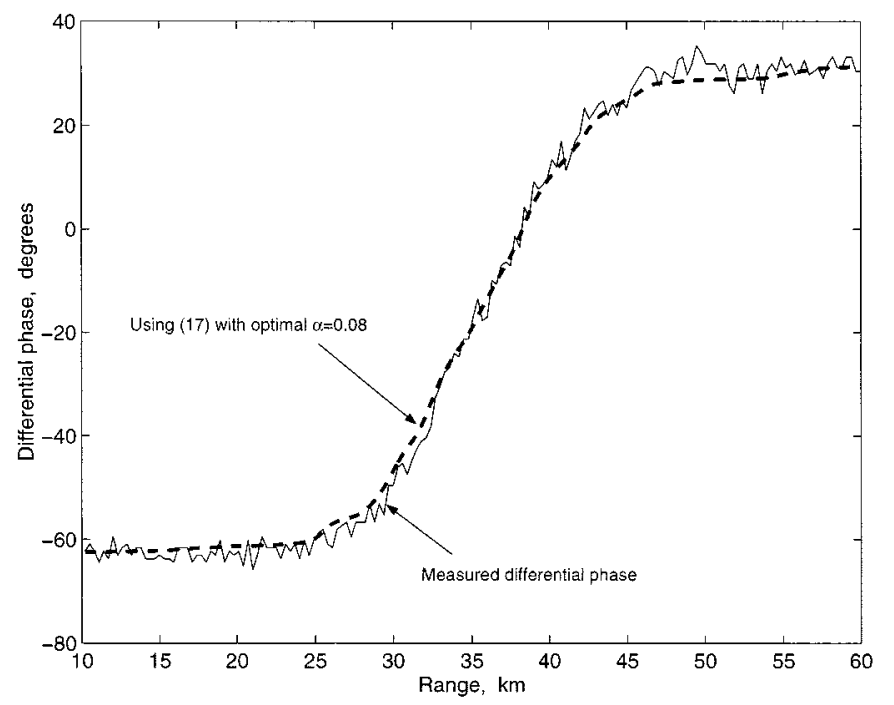

(c)

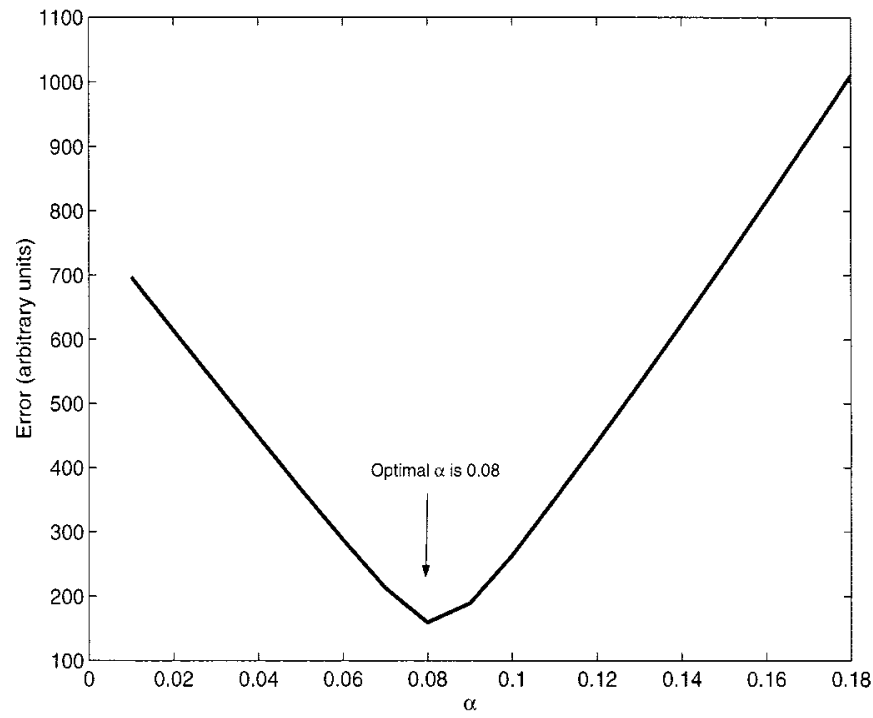

(b)

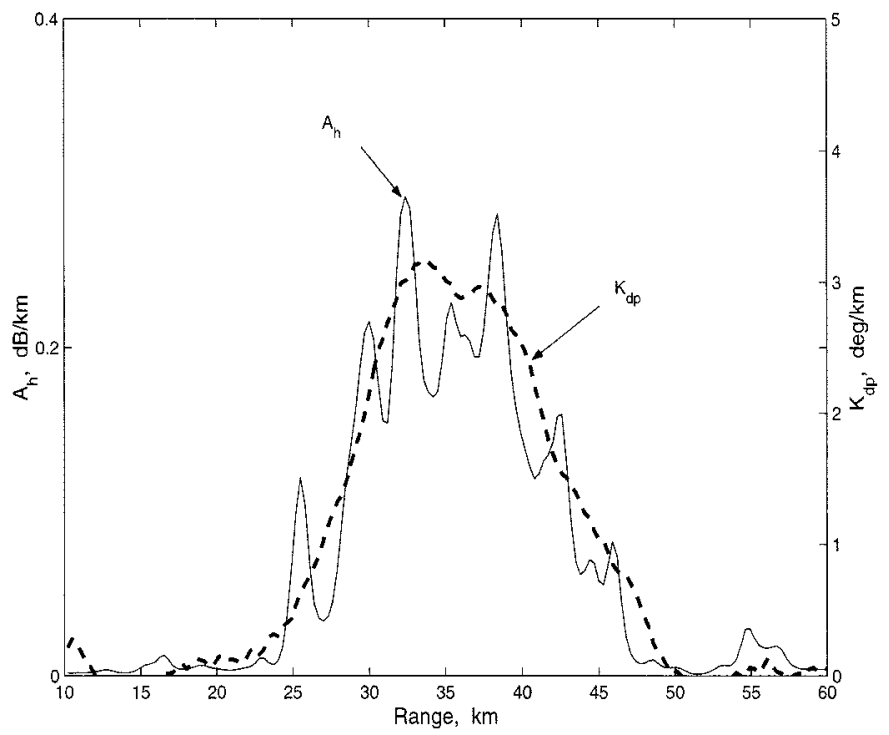

(d)

Fig. 4 (a) Constructed $\Phi_{\mathrm{dp}}$ range profiles and the filtered $\Phi_{\mathrm{clp}}$ profile. (b) The error in (18) versus $\alpha$. (c) Comparing the measured $\Phi_{\mathrm{clp}}$ with constructed $\Phi_{\mathrm{clp}}$ using the optimal $\alpha$. (d) Retrieved specific attenuation range profile and the $K_{\mathrm{dp}}$ from the filtered $\Phi_{\mathrm{cp}}$.

Note that the standard ZPHI method with an a priori fixed value of $\alpha$ (quoted as 0.113 in [8] for C-band) would only constrain the final $\Phi_{\mathrm{dp}}$ value, whereas the iterative method with an optimal $\alpha$ would tend to obtain the "best" $A_{h}(r)$ profile such that the constructed $\Phi_{\mathrm{dp}}^{c}$ would agree with the measured $\Phi_{\mathrm{dp}}$ over the entire range, as illustrated in Fig. 4(c). This self-consistent procedure is an important extension to the standard ZPHI method since $\alpha$ is temperature-dependent (varying by a factor of 2 at $\mathrm{C}$-band in the temperature range $0-30{ }^{\circ} \mathrm{C}$ [17]), as well as being inversely dependent on the slope $\gamma$ (varying by a factor of 2 for $\gamma$ in the range $0.04-0.08$ [25]). In fact, it is concluded in [8] that ... "A critical aspect of ZPHI is the choice of the oblateness law for raindrops" which simply relates to choosing an a priori fixed value for $\alpha$. The optimal choice of $\alpha$ tends to force $A_{h}(r)$ to follow $K_{\mathrm{dp}}(r)$ on average, as illustrated in Fig. 4(d), and this tends to mitigate the constant $N_{o}$ assumption of the standard ZPHI method. From theory, $A_{h}(r)$ is linearly related to $K_{\mathrm{dp}}$ at C-band and the coefficient $\alpha$ does not depend on $N_{o}$.

In practice, an optimal $\alpha$ which minimizes the error in (18) may not always exist, particularly when $\Delta \Phi_{\mathrm{dp}}\left(r_{o} ; r_{m}\right) \leq 30^{\circ}$, in which case the most likely value in the range $\left(\alpha_{\min }, \alpha_{\max }\right)$ may be chosen (i.e., $\alpha=0.08$ ). The probability of finding an optimal $\alpha$ increases substantially when $\Phi_{\mathrm{dp}}\left(r_{o} ; r_{m}\right)>30^{\circ}$ and this threshold is used in this paper. This method is particularly suitable for strong rain cells for which accurate attenuation-correction is most needed.

The retrieval of the range profile of specific attenuation, $A_{h}(r)$, is valuable in its own right [see Fig. 4(d)] since it can be related to rain rate $R$ by a power law similar to $R\left(K_{\mathrm{dp}}\right)$ algorithms. It is clear from (14) that $A_{h}$ is independent of the radar system constant [8]. However, it has two other advantages as compared with $R\left(K_{\mathrm{dp}}\right)$, namely, 1) the optimal $\alpha$ determination avoids the use of a fixed value for $\gamma$ and 2) 
$A_{h}$ estimates are available at the basic range resolution of the radar, unlike $K_{\mathrm{dp}}$ estimates which, of necessity, are based on a range smoothing interval (in practice, $\Phi_{\mathrm{dp}}$ data from 10 to 20 consecutive range resolution volumes are used [26]).

\section{Self-Consistent Method For Correction of $Z_{\mathrm{dr}}$}

The specific differential attenuation $\left(A_{\mathrm{dp}}=A_{h}-A_{v}, \mathrm{~dB}\right.$ $\mathrm{km}^{-1}$ ) profile must be first estimated before correcting the measured $Z_{\mathrm{dr}}^{\prime}$. Similar to (16), the correction equation can be written as

$$
Z_{\mathrm{dr}}(r)=Z_{\mathrm{dr}}^{\prime}(r)+2 \int_{r_{o}}^{r} A_{\mathrm{dp}}(s) d s .
$$

While the linear relation $A_{\mathrm{dp}}=\beta K_{\mathrm{dp}}$ with fixed $\beta$ was suggested in[6] and [7], as a method of correction, no constraint was imposed on the final value of $Z_{\mathrm{dr}}$ at the range $r_{m}$ on the far side of the rain cell where presumably $Z_{\mathrm{dr}}$ tends to $0 \mathrm{~dB}$ because small (and nearly spherical) drops are expected (see Fig. 2). Use of a priori fixed $\beta$ and no constraint on $Z_{\mathrm{dr}}\left(r_{m}\right)$ is a drawback of this simple method. Simulations show that the $A_{\mathrm{dp}}-K_{\mathrm{dp}}$ relation is not quite linear with the exponent (at $\mathrm{C}$-band) varying between 1.18 and 1.25 [17].

To overcome this problem, a constraint-based method was proposed in [9], where the cumulative effects of differential attenuation caused by strong rain cells along the propagation path frequently result in $Z_{\mathrm{dr}}^{\prime}\left(r_{m}\right)$ to be negative. By assumption, the intrinsic $Z_{\mathrm{dr}}\left(r_{m}\right)$ is set to $0 \mathrm{~dB}$ (i.e., spherical drops), and, thus, the path-integrated differential attenuation is $\Delta Z_{\mathrm{dr}}\left(r_{m}\right)$

$$
\begin{aligned}
\Delta Z_{\mathrm{dr}}\left(r_{m}\right) & =Z_{\mathrm{dr}}^{\prime}\left(r_{m}\right)-Z_{\mathrm{dr}}\left(r_{m}\right) \\
& \approx Z_{\mathrm{dr}}^{\prime}\left(r_{m}\right) \\
& =-\beta\left[\Phi_{\mathrm{dp}}\left(r_{m}\right)-\Phi_{\mathrm{dp}}\left(r_{o}\right)\right] .
\end{aligned}
$$

An estimate of $\beta$ can then be obtained as

$$
\hat{\beta}=\frac{\left|Z_{\mathrm{dr}}^{\prime}\left(r_{m}\right)\right|}{\left[\Phi_{\mathrm{dp}}\left(r_{m}\right)-\Phi_{\mathrm{dp}}\left(r_{o}\right)\right]} .
$$

The specific differential attenuation $A_{\mathrm{dp}}(r)$ at each range location along the propagation path can be estimated as

$$
\begin{aligned}
\hat{A}_{\mathrm{dp}}(r) & =\hat{\beta} K_{\mathrm{dp}}(r) \\
& =\frac{\left|Z_{\mathrm{dr}}^{\prime}\left(r_{m}\right)\right|}{\left[\Phi_{\mathrm{dp}}\left(r_{m}\right)-\Phi_{\mathrm{dp}}\left(r_{o}\right)\right]} K_{\mathrm{dp}}(r) .
\end{aligned}
$$

Note that $\hat{\beta}$ can vary from beam to beam in this method and an a priori value need not be assumed. In essence, this method of correcting the measured $Z_{\mathrm{dr}}^{\prime}$ uses the constraint that the intrinsic $Z_{\mathrm{dr}}$ on the far side of an intense rain cell should tend to 0 $\mathrm{dB}$, representative of light drizzle conditions. If this constraint cannot be established, the $\hat{\beta}$ must be estimated as

$$
\hat{\beta}=\frac{\left|Z_{\mathrm{dr}}^{\prime}\left(r_{m}\right)-Z_{\mathrm{dr}}\left(r_{m}\right)\right|}{\left[\Phi_{\mathrm{dp}}\left(r_{m}\right)-\Phi_{\mathrm{dp}}\left(r_{o}\right)\right]}=\frac{\left|\Delta Z_{\mathrm{dr}}\left(r_{m}\right)\right|}{\left[\Phi_{\mathrm{dp}}\left(r_{m}\right)-\Phi_{\mathrm{dp}}\left(r_{o}\right)\right]}
$$

where the intrinsic value of $Z_{\mathrm{dr}}\left(r_{m}\right)$ must be established by other physical constraints. Note that $\Delta Z_{\mathrm{dr}}\left(r_{m}\right)=Z_{\mathrm{dr}}^{\prime}\left(r_{m}\right)-$ $Z_{\mathrm{dr}}\left(r_{m}\right)$ must be negative (in decibel units) in (25) because oblate raindrops can only cause positive $A_{\mathrm{dp}}$ (or $A_{h}>A_{v}$ ).
The self-consistent method for correcting $Z_{\mathrm{dr}}$ proposed in this paper assumes that the measured $Z_{\mathrm{dr}}^{\prime}$ has first been corrected by the self-consistent ZPHI method. Thus, the intrinsic value of $Z_{h}\left(r_{m}\right)$ is available on the far side of the rain cell. For the purposes of this method, it is assumed that $Z_{\mathrm{dr}}\left(r_{m}\right)$ can be estimated from $Z_{h}\left(r_{m}\right)$ at least in an average sense. This average relationship can be based on scattering simulations using either measured drop size distributions or assuming a gamma model for $N(D)$, Here, $N(D)$ measured by a Joss-Waldvogel disdrometer [27] during SCSMEX were used to arrive at

$$
\begin{aligned}
& \bar{Z}_{\mathrm{dr}}\left(r_{m}\right) \\
& \quad= \begin{cases}0 ; & \bar{Z}_{h}\left(r_{m}\right) \leq 20 \mathrm{dBZ} \\
0.048 \bar{Z}_{h}\left(r_{m}\right)-0.774 ; & 20<\bar{Z}_{h}\left(r_{m}\right) \leq 45 \mathrm{dBZ}\end{cases}
\end{aligned}
$$

which is valid at C-band. The raindrop model assumes oblate shapes with axis ratios given in [14] for $D<4 \mathrm{~mm}$ and as given in [13] for $D>4 \mathrm{~mm}$; a Gaussian distribution of canting angles with zero mean and standard deviation $10^{\circ}$ (see [22, Ch. 7] for details on the simulation procedure) is also assumed. The overbar on $Z_{\mathrm{dr}}$ and $Z_{h}$ refers to average values; note that the units of $\bar{Z}_{h}$ are in dBZ in (26).

Since the correction procedure is performed along a single beam of data, it is possible to manually examine such data and to preselect $r_{m}$ on the far side of a rain cell where $\bar{Z}_{h}\left(r_{m}\right)<20$ $\mathrm{dBZ}$ and $Z_{\mathrm{dr}}\left(r_{m}\right)=0 \mathrm{~dB}$. For real-time application, the $r_{m}$ must be automatically determined for each beam based on an algorithm that detects the end of the "good" data segment on the far side of a rain cell (see [28, Appendix A]). Generally, the $Z_{h}$ drops off rapidly beyond $r_{m}$ so the $\bar{Z}_{h}\left(r_{m}\right)<45 \mathrm{dBZ}$, and $\bar{Z}_{\mathrm{dr}}\left(r_{m}\right)$ can be estimated using (26). If multiple rain cells are present along the beam, the algorithm can be applied to each rain cell by appropriate partitioning of the cells along the range.

The correction method starts with the first estimate of the $A_{\mathrm{dp}}$ range profile by assuming that it is linearly related to $A_{h}\left(r ; \alpha_{\mathrm{opt}}\right)$

$$
A_{\mathrm{dp}}(r ; \beta)=\frac{\beta}{\alpha_{\mathrm{opt}}} A_{h}\left(r ; \alpha_{\mathrm{opt}}\right) .
$$

Recall that $A_{h}\left(r ; \alpha_{\text {opt }}\right)$ is assumed to be obtained first using the self-consistent ZPHI method. The initial value of $\beta$ is obtained from (25), and (27) is used in (19) to arrive at the first estimate of the range profile of $Z_{\mathrm{dr}}(r ; \beta)$. The occurrence of "giant" raindrops along the beam are detected using the measurement of backscatter differential phase [23] and the $A_{\mathrm{dp}}$ is increased locally using the empirical scheme suggested in [19]. Using this first guess of $A_{\mathrm{dp}}$, the first estimate of $\hat{Z}_{\mathrm{dr}}\left(r_{m} ; \beta\right)$ is calculated using (19)

$$
\hat{Z}_{\mathrm{dr}}\left(r_{m} ; \beta\right)=Z_{\mathrm{dr}}^{\prime}\left(r_{m}\right)+\frac{2 \beta}{\alpha_{\mathrm{opt}}} \int_{r_{0}}^{r_{m}} A_{h}\left(s ; \alpha_{\mathrm{opt}}\right) d s .
$$

This first estimate of $\hat{Z}_{\mathrm{dr}}\left(r_{m} ; \beta\right)$ is compared to the constraint value $\bar{Z}_{\mathrm{dr}}\left(r_{m}\right)$ determined earlier [see (26)]. If $\hat{Z}_{\mathrm{dr}}\left(r_{m} ; \beta\right)$ is larger than the constraint value, then the cumulative differential attenuation was over-predicted and the next value of $\beta$ in (27) is adjusted to a lower value and the steps [(27) and (28)] are repeated until an optimal $\beta$ is found that results in 


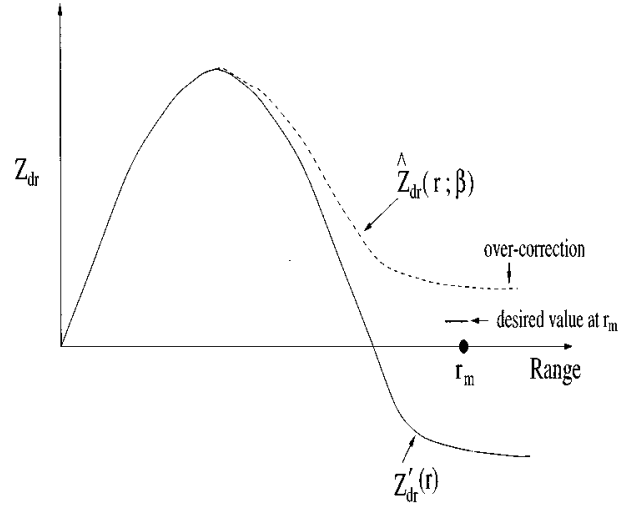

Fig. 5. Illustration relative to estimating the optimal $\beta$ in (28).

$\hat{Z}_{\mathrm{dr}}\left(r_{m} ; \beta\right)-\bar{Z}_{\mathrm{dr}}\left(r_{m}\right)$ being less than a preselected tolerance (e.g., $0.2 \mathrm{~dB}$ ). If the cumulative differential attenuation is under-predicted $\left(\hat{Z}_{\mathrm{dr}}\left(r_{m} ; \beta\right)<\bar{Z}_{\mathrm{dr}}\left(r_{m}\right)\right)$, then the $\beta$ value may be successively increased in (27) until an optimal $\beta$ is found. Fig. 5 schematically illustrates this adjustment procedure for the case where the initial $\beta$ leads to "over correction." Once the optimal $\beta$ is estimated, the final corrected $Z_{\mathrm{dr}}$ at each range location is obtained from

$$
\hat{Z}_{\mathrm{dr}}\left(r ; \beta_{\mathrm{opt}}\right)=Z_{\mathrm{dr}}^{\prime}(r)+2 \frac{\beta_{\mathrm{opt}}}{\alpha_{\mathrm{opt}}} \int_{r_{0}}^{r} A_{h}\left(s ; \alpha_{\mathrm{opt}}\right) d s .
$$

The corrected $Z_{\mathrm{dr}}$ using this method is shown in Fig. 3(b).

\section{C-POL Radar Data ANALYSis From SCSMEX}

Attenuation-correction procedures are difficult to validate without coordinated independent measurements, e.g., rain rate from gages $N(D)$ from disdrometers. A few previous studies have attempted to validate their correction procedures by comparing the rain rate from corrected $Z_{h}$ and/or $Z_{\mathrm{dr}}$ data with rain gages [4], [9], [18]. However, most studies use the corrected radar data itself to establish internal validity, for example, by plotting $K_{d p}$ against corrected and uncorrected $Z_{h}$ (and $Z_{\mathrm{dr}}$ ) [9], [18], [19]. Note that $K_{\mathrm{dp}}$ is unaffected by attenuation and thereby forms a reference axis for internal validity.

The C-POL radar operated by the Australian Bureau of Meteorology Research Center is described in [24]. It is normally located in Darwin, N. Territory, but was moved in the summer of 1998 to Dongsha Island $\left(20^{\circ} 42^{\prime} \mathrm{N}, 116^{\circ} 43^{\prime} \mathrm{E}\right)$ in the South China Sea. Raindrop size distributions (over 800 2-min averaged distributions) were available at this location from a JossWaldvogel disdrometer [27] which was operated for two months (May 1-June 30,1998). Scattering simulations using these measured $N(D)$ were performed to yield $Z_{h}, Z_{\mathrm{dr}}$, and $K_{\mathrm{dp}}$ data which are considered representative for the rain types occurring in this region. Details of the scattering simulation procedure are given in [22, Ch. 7].

C-POL radar date from a typical convective rain cell on May 18, 1998 were corrected for attenuation and differential attenuation using the self-consistent methods described previously. These radar data $\left(Z_{h}, Z_{h}^{\prime}, Z_{\mathrm{dr}}, Z_{\mathrm{dr}}^{\prime}, K_{\mathrm{dp}}, A_{h}\right.$, and $\left.A_{\mathrm{dp}}\right)$ are available at each range resolution volume (spaced $300 \mathrm{~m}$ apart) as the radar scanned this rain cell; a typical beam of data was shown earlier in Figs. 3 and 4. Data from over 14000 resolution volumes are available spanning a wide range of rain intensities, primarily of the convective type. The system gain for the C-POL radar was based on solar calibrations made several times during SCSMEX. ${ }^{1}$ The $Z_{\mathrm{dr}}$ system offset was based on vertically pointing the antenna in rain and rotating the antenna in azimuth $360^{\circ}$ (see [22, Ch. 7]).

Fig. 6(a) shows scatter plots of $K_{\mathrm{dp}}$ versus uncorrected $Z_{h}$ and corrected $Z_{h}$ from the radar data, as well as from scattering simulations based on disdrometer data. The optimal $\alpha$ values for each beam of data range from 0.04 to 0.135 . Note how the corrected $Z_{h}$ data, on average, follow the disdrometer-based simulations more closely, especially for $Z_{h}>45 \mathrm{dBZ}$, i.e., stronger rain rate events. Even though the radar data exhibit considerable scatter about the simulation curve, the importance of accurate correction of $Z_{h}$ is especially evident at higher reflectivities. It is noted that this internal validity check does not necessarily prove that the correction scheme proposed herein is necessarily superior to the standard ZPHI method or to other attenuation correction methods [8], [18], [19].

Fig. 6(b) shows similar scatter plots of $K_{\mathrm{dp}}$ versus $Z_{\mathrm{dr}}$. The importance of $Z_{\mathrm{dr}}$ correction is more evident in this figure, and over nearly the entire range of $K_{\mathrm{dp}}$ values. While the radar data show considerable scatter, the corrected $Z_{\mathrm{dr}}$ data are more closely aligned with the disdrometer-based simulations, especially, the nearly steady value of corrected $Z_{\mathrm{dr}}(1-1.5 \mathrm{~dB})$ for $K_{\mathrm{dp}}>3^{\circ} \mathrm{km}^{-1}$. On physical grounds, this is representative of "equilibrium" drop size distributions which occur when there is a balance between the processes of drop breakup and drop coalescence at high rain rates that leads to a steady value of the median volume diameter $D_{o}$ in (10) (and represented by the narrow range in $Z_{\mathrm{dr}}$ values) [22, Ch. 7], [29].

Fig. 6(c) shows the scatter plots of $Z_{\mathrm{dr}}$ versus $Z_{h}$ which again illustrates the necessity of attenuation-correction, and the excellent agreement of the corrected data with the disdrometer-based simulation.

Because self-consistent methods described herein yield the specific attenuation $\left(A_{h}\right)$ and specific differential attenuation $\left(A_{\mathrm{dp}}\right)$ at each resolution volume, it is useful to validate these retrievals by comparing them against disdrometer-based scattering simulations. Recall from (11) that a power law relation is assumed to exist between $A_{h}$ and $Z_{h}$; however, the retrieval of $A_{h}$ is not dependent on the multiplicative coefficient $a$ in (11) but only on the exponent $b$ ( $b$ is fixed at 0.78 at C-band, which is obtained from disdrometer-based scattering simulations). Fig. 7(a) shows the scatter plot of $A_{h}$ versus corrected $Z_{h}$ which is compared against disdrometer-based scattering simulations. The agreement between the two, on average, serves as an additional internal validity check on the retrieval of $A_{h}$ and on the correction of $Z_{h}$. A similar scatter plot of $A_{\mathrm{dp}}$ versus $A_{h}$ is shown in Fig. 7(b), and again the agreement, on average, is excellent, especially for $Z_{h}>45 \mathrm{dBZ}$. This scatter plot is considered a more stringent test of the retrieval of $A_{\mathrm{dp}}$ and the $Z_{h}$ correction methodology than that shown in Fig. 7(a).

\footnotetext{
${ }^{1}$ See
} smex_radar 


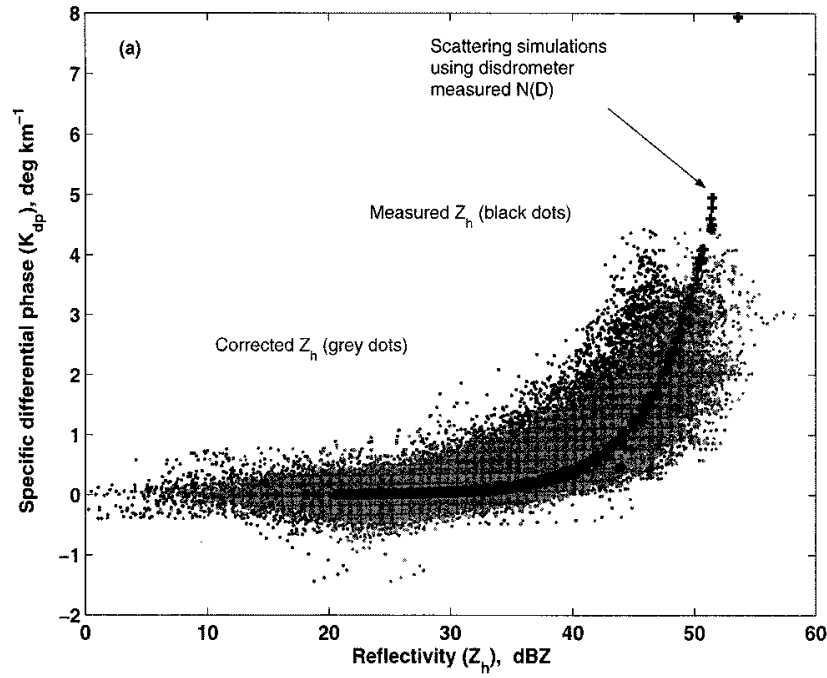

(a)

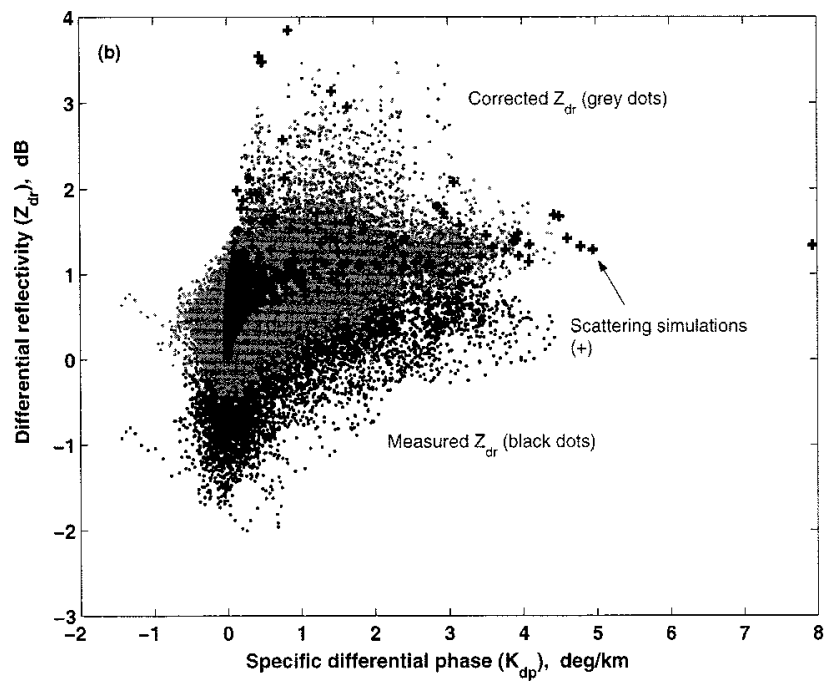

(b)

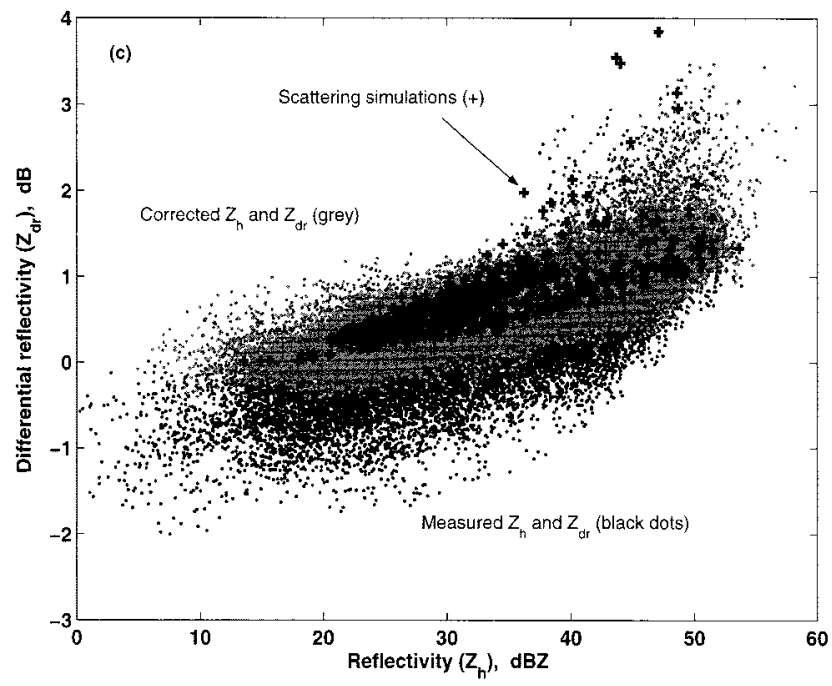

(c)

Fig. 6 (a) Scatter plot of $K_{\mathrm{clp}}$ versus $Z_{h}$ (both corrected and uncorrected) using C-POL radar data from one rain cell in SCSMEX. Scattering simulations using disdrometer-measured drop size distribution from SCSMEX are also shown. (b) Same as (a) except $Z_{\mathrm{dr}}$ versus $K_{\mathrm{dp}}$ is shown. (c) Same as (a) except $Z_{\mathrm{dr}}$ versus $Z_{h}$ is shown.

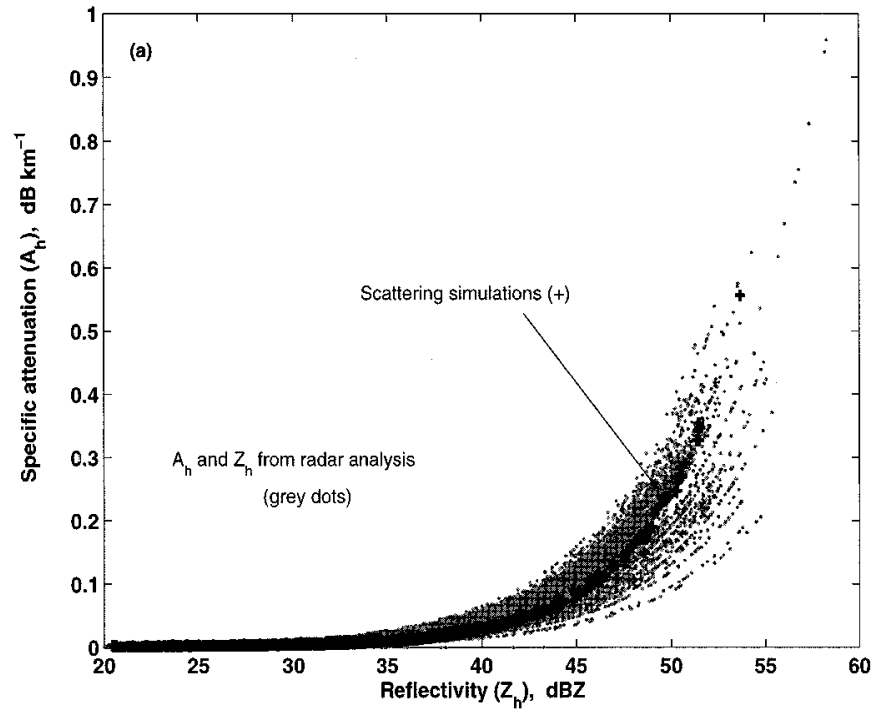

(a)

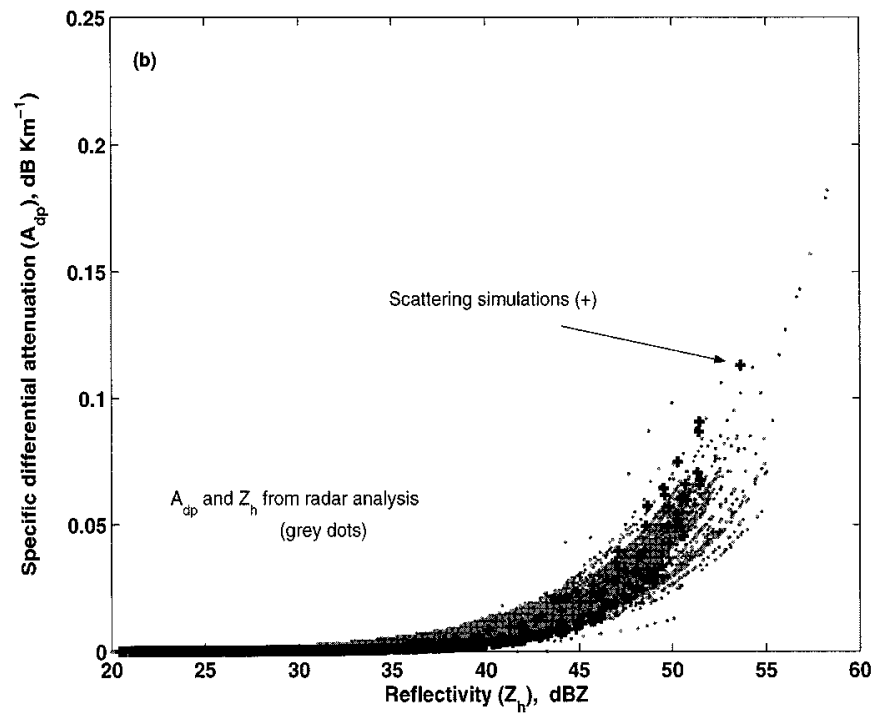

(b)

Fig. 7 (a) Scatter plot of the retrieved $A_{h}$ versus corrected $Z_{h}$ from radar data. Also, a similar plot based on disdrometer-measured drop size distributions from SCSMEX. (b) Same as (a) except $A_{\mathrm{dp}}$ versus corrected $Z_{h_{t}}$ from radar data compared to simulations.

One important application of the corrected $Z_{\mathrm{dr}}$ data is the retrieval of the median volume diameter $D_{o}$. For equilibrium drop axis ratios and gamma form of $N(D)$, it is possible to relate $Z_{\mathrm{dr}}$ to $D_{o}$ via a power law of the form $D_{o}=a Z_{\mathrm{dr}}^{b}$ (see [22, Ch. 7] or [30]). However, the power law coefficients depend on the slope $\gamma$ of the relation between the drop axis ratio $(r)$ and drop diameter ( $r=1-\gamma D ; D$ in millimeters) which can change due to drop oscillations [14]. A more robust retrieval of $D_{o}$, which is relatively immune to change in $\gamma$, depends on first estimating $\gamma$ from radar measurements of $Z_{h}, Z_{\mathrm{dr}}$, and $K_{\mathrm{dp}}$ [10]. At C-band, both $Z_{h}$ and $Z_{\mathrm{dr}}$ must first be accurately corrected for attenuation before $\gamma$ can be estimated. The algorithm for estimating $\gamma$ at C-band is based on simulations described in [10]

$$
\gamma=2.36 \cdot 10^{-0.0398 Z_{h_{h}}} \cdot 10^{0.066 Z_{\mathrm{dr}}} \cdot K_{\mathrm{dp}}^{0.426}
$$



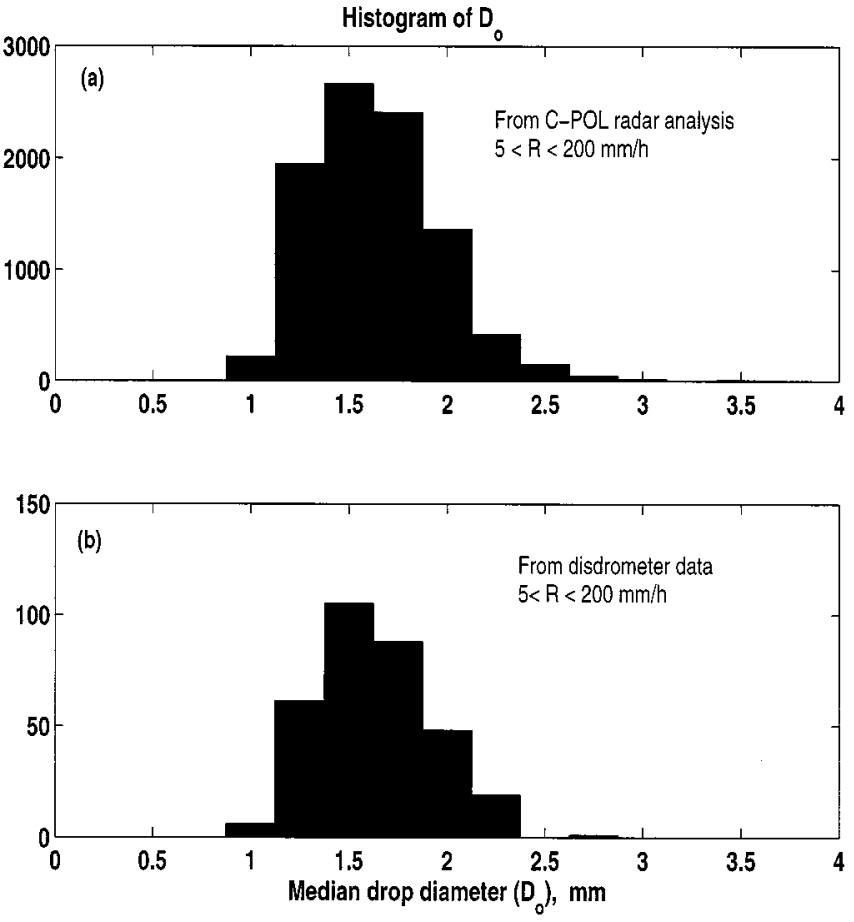

Fig. 8. (a) Histogram of $D_{o}$ from radar data using (31). (b) Histogram of $D_{o}$ from disdrometer-measured drop size distributions.

where $Z_{h}$ is in units of dBZ, $Z_{\mathrm{dr}}$ is in $\mathrm{dB}$, and $K_{\mathrm{dp}}$ is in ${ }^{\circ} \mathrm{km}^{-1}$. The retrieval of $D_{o}$ is again based on simulations described in [25]

$$
D_{o}=a\left[10^{0.1\left(Z_{h}\right)}\right]^{b}\left[10^{0.1\left(Z_{\mathrm{dr}}\right)}\right]^{c}
$$

where

$$
\begin{aligned}
a & =0.571(\gamma)^{0.027} \\
b & =0.0587(\gamma)^{-0.168} \\
c & =0.0526(\gamma)^{-0.808}
\end{aligned}
$$

The histogram of $D_{o}$ retrieved using (30), (31), and the corrected C-POL radar data (same radar data used in the scatter plots in Fig. 6) is shown in Fig. 8(a). Only data points where the radar-estimated rain rate $>5 \mathrm{~mm} / \mathrm{h}^{-1}$ are used in constructing the histogram, e.g., to be representative of convective rainfall. Fig. 8(b) shows the histogram of $D_{o}$ from disdrometer measurements [same $N(D)$ data that were used in the disdrometer-based scattering simulations in Figs. 6 and 7]; again, only data with $R>5 \mathrm{~mm} / \mathrm{h}^{-1}$ were used in constructing the histogram. Since the disdrometer data are strongly weighted by stratiform rain events (because of their long duration relative to convective events), a rain rate threshold of $5 \mathrm{~mm} / \mathrm{h}^{-1}$ is applied before the histograms are compared. This threshold generally ensures that primarily convective rain types are included in the histogram based on disdrometer data. While comparing the two histograms, it is important to keep in mind that the radar data were obtained from scanning one convective rain cell whereas the disdrometer data are based on two months of collection in a variety of rain types (restricting $R>5 \mathrm{~mm} / \mathrm{h}^{-1}$ tends to narrow the rain types to primarily convective). The similarity in shape of the two histograms is quite remarkable, in particular, the mode is near $1.5 \mathrm{~mm}$. While such histogram comparisons reinforce the accuracy of the self-consistent correction schemes proposed here, the radar-based retrieval of $D_{o}$ is expected to be useful in improving cloud resolving models that can predict $D_{o}$ using "bulk" microphysical schemes, or in improving satellite-based radar or microwave techniques for estimating surface rainfall.

\section{SUMMARY AND CONCLUSIONS}

There is no doubt that the measurement of $\Phi_{\mathrm{dp}}$ by a dual-polarized radar serves to "stabilize" attenuation-correction algorithms proposed recently [6], [18], [19], as compared to earlier algorithms (e.g., [2]-[4]) which did not use $\Phi_{\mathrm{dp}}$. The introduction of constraints in attenuation-correction algorithms in an important advance, and in this paper a self-consistent scheme has been proposed and evaluated that extends the previous work in [8], and [9] in several important respects. First, the $\alpha$ value in $A_{h}=\alpha K_{\mathrm{dp}}$ is not assumed a priori, but is determined via a minimization process for each beam of radar data. It is known that $\alpha$ is not only temperature-dependent, but also varies with the drop axis ratio versus $D$ relation. Thus, the scheme proposed herein overcomes these two potential limitations of the standard ZPHI method [8] in an "average" sense by determining an optimal $\alpha\left(\alpha_{\text {opt }}\right)$ for each beam. The comparisons of the derived $A_{h}$ versus corrected $Z_{h}$ is in excellent agreement with disdrometer-based scattering simulations. Such comparisons as well as others are used to validate, in a self-consistent manner, the accuracy of the attenuation-correction scheme. The retrieval of $A_{h}$ at each resolution volume also allows the estimation of rain rate without the smoothing problem inherent in $K_{\mathrm{dp}}$-based estimates of $R$.

Correction of $Z_{\mathrm{dr}}$ is also based on a self-consistent scheme with constraints and avoids the assumption of a constant $\beta$ value in $A_{\mathrm{dp}}=\beta K_{\mathrm{dp}}$. The approach here differs from [9] in two respects. First, it is assumed that $A_{\mathrm{dp}}$ is linearly related to $A_{h}$ [i.e., $A_{\mathrm{dp}}=\left(\beta / \alpha_{\mathrm{opt}}\right) A_{h}$ ] which is a good approximation at C-band, and that $A_{h}$ is obtained as the first step with an optimal $\alpha$. Thus, the $Z_{h}\left(r_{m}\right)$ on the far side of the rain cell is known, and in rain, an average value of $Z_{\mathrm{dr}}\left(r_{m}\right)$ can be established (which is the desired value). Note that it is not necessary to assume that $Z_{\mathrm{dr}}\left(r_{m}\right)$ is $0 \mathrm{~dB}$ in this scheme. The resulting advantage is that the $r_{m}$ need not be selected manually for each beam of data, rather an algorithm is used to estimate $r_{m}$ (or, the end of the "good" data segment on the far side of the rain cell). It follows that $Z_{h}\left(r_{m}\right)$ and $Z_{\mathrm{dr}}\left(r_{m}\right)$ can be constrained on average in a self-consistent way on the far side of the rain cell, without assuming that the drops are spherical there. Second, the $\beta$ value is iteratively adjusted so that the corrected $Z_{\mathrm{dr}}\left(r_{m}\right)$ is close to the desired $Z_{\mathrm{dr}}\left(r_{m}\right)$ within a prescribed tolerance. The comparison of the retrieved $A_{\mathrm{dp}}$ versus corrected $Z_{h}$ is found to be in excellent agreement with disdrometer-based scattering simulations. These as well as other such comparisons are used to validate the attenuation-correction schemes proposed herein.

One quantitative application of the corrected $Z_{h}$ and $Z_{\mathrm{dr}}$ radar data is the retrieval of $D_{o}$ of the $N(D)$. A new algorithm is used to retrieve $D_{o}$ based on $Z_{h}, Z_{\mathrm{dr}}$, and $K_{\mathrm{dp}}$ that is relatively immune to the drop axis ratio versus $D$ relation [10], [25]. This retrieval is validated by comparing the shape of the histogram of $D_{o}$ obtained from $N(D)$ measured by a 
disdrometer of a two-month period. The remarkable agreement in the shapes of these two histograms lends further validity to the attenuation-correction schemes proposed in this paper. Indeed, the accurate retrieval of $D_{o}$ from C-band radar measurements is generally considered a difficult problem, and the methods proposed here constitute an important advance in the quantitative application of C-band radar in the remote determination of rain microphysics.

\section{ACKNOWLEDGMENT}

The authors would like to thank Colorado State University and the BMRC for their support. They would also like to thank Dr. E. Gorgucci who performed the C-band simulations resulting in (30) and (31). The authors acknowledge the C-POL radar staff for their dedicated operation of the radar during SCSMEX.

\section{REFERENCES}

[1] PH. Hildebrand, "Iterative correction for attenuation of $5 \mathrm{~cm}$ radar in rain," J. Appl. Meteor, vol. 17, pp. 508-514, 1978.

[2] R. Meneghini, "Rain rate estimates for an attenuating radar," Radio Sci., vol. 13, pp. 459-470, 1978

[3] K. Aydin, Y. Zhao, and T. A. Seliga, "Rain-induced attenuation effects on C-band dual-polrization meteorological radar," IEEE Trans. Geosci. Remote Sensing, vol. 27, pp. 57-66, Jan. 1989.

[4] E. Gorgucci, G. Scarchilli, and V. Chandrasekar, "Error structure of radar rainfall measurement at $\mathrm{C}$-band frequencies with dual-polarization algorithm for attenuation correction," J. Geophys. Res., vol. 101, pp. 26461-26471, 1996

[5] R. Meneghini, K. Nakamura, C. W. Ulbrich, and D. Atlas, "Experimental test of methods for the measurement of rainfall using an airborne dualwavelength radar," J. Atmos. Ocean. Technol., vol. 6, pp. 637-651, 1989.

[6] V. N. Bringi, V. Chandrasekar, N. Balakrishnan, and D. S. Zrnić, "An examination of propagation effects in rainfall on radar measurements at microwave frequencies," J. Atmos. Ocean. Technol., vol. 7, pp. 829-840, 1990.

[7] A. R. Holt, "Extraction of differential propagation phase from data from S-band circularly polarized radars," Electron. Lett., vol. 24, pp. $1241-1242,1988$

[8] J. Testud, E. Le Bouar, E. Obligis, and M. Ali-Mehenni, "The rain profiling algorithm applied to polarimetric weather radar," J. Atmos. Ocean. Technol., vol. 17, pp. 322-356, 2000

[9] T. J. Smyth and A. J. Illingworth, "Correction for attenuation of radar reflectivity using polarizatin data," Q. J. R. Meteorol. Soc., vol. 124, pp. 2393-2415, 1998.

[10] E. Gorgucci, G. Scarchilli, V. Chandrasekar, and V. N. Bringi, "Measurement of mean raindrop shape from polarimetric radar observations," $J$. Atmos. Sci., vol. 57, pp. 3406-3413, 2000.

[11] H. C. van de Hulst, Light Scattering by Small Particles. New York: Dover, 1981.

[12] D. Atlas and C. W. Ulbrich, "Path- and area-integrated rainfall measurement by microwave attenuation in 1-3 cm band," J. Appl. Meteor., vol. 16, pp. 1322-1331, 1977

[13] K. V. Beard and C. Chuang, "A new model for the equalibrium shape of raindrops," J. Atmos. Sci., vol. 44, pp. 1509-1524, 1987.

[14] K. Andsager, K. V. Beard, and N. F. Laird, "Laboratory measurements of axis ratios for large raindrops," J. Atmos. Sci., vol. 56, pp. 2673-2683, 1999.

[15] H. R. Pruppacher and K. V. Beard, "A wind tunnel investigation of the internal circulation and shape of water drops falling at terminal velocity in air," Q. J. R. Meteorol. Soc., vol. 96, pp. 247-256, 1970.

[16] A. R. Jameson, "Microphysical interpretation of multi-parameter radar measurements in rain. Part III: Interpretation and measurement of propagation differential phase shift between orthogonal linear polarizations,' J. Atmos. Sci., vol. 42, pp. 607-614, 1985.

[17] — "The effect of temperature on attenuation correction schemes in rain using polarization propagation differential phase shift," J. Appl. Meteor., vol. 31, pp. 1106-1118, 1992.
[18] A. Ryzhkov and D. S. Zrnić, "Precipitation and attenuation measurements at $10 \mathrm{~cm}$ wavelength," J. Appl. Meteor., vol. 34, pp. 2121-2134, 1995.

[19] L. D. Carey, S. A. Rutledge, D. A. Ahijevych, and T. D. Keenan, "Correcting propagation effects in C-band polarimetric radar observations of tropical convection using differential propagation phase," J. Appl. Meteor., vol. 39, pp. 1405-1433, 2000.

[20] M. Marzoug and P. Amayenc, "A class of single and dual frequency algorithms for rain-rate profiling from a spaceborne radar, Part I: Principles and tests from numerical simulations," J. Atmos. Ocean. Technol., vol. 11, pp. 1480-1506, 1994.

[21] W. F. Hitschfeld and J. Bordan, "Errors inherent in the radar measurement of rainfall at attenuating wavelengths," J. Meteor., vol. 11, pp. $58-67,1954$

[22] V. N. Bringi and V. Chandrasekar, Polarimetric Doppler Weather Radar: Principles and Applications. Cambridge, U.K.: Cambridge Univ. Press, 2000.

[23] J. Hubbert and V. N. Bringi, "An iterative filtering technique for the analysis of copolar differential phase and dual-frequency radar measurements," J. Atmos. Ocean. Technol., vol. 12, pp. 643-648, 1995.

[24] T. Keenan, K. Glasson, F. Cummings, T. S. Bird, R. J. Keeler, and J. Lutz, "The BMRC/NCAR C-band polarimetric (C-Pol) radar system," J. Atmos. Ocean. Technol., vol. 15, pp. 871-886, 1998.

[25] E. Gorgucci, G. Scarchilli, and V. Chandrasekar, "Rainfall estimation from polarimetric radar measurements: Composite algorithms independent of raindrop shape-size relation," J. Atmos. Ocean. Technol., to be published.

[26] E. Gorgucci, G. Scarchilli, and V. Chandrasekar, "Specific differential phase shift estimation in the presence of nonuniform rainfall medium along the path," J. Atmos. Ocean. Technol., vol. 16, pp. 1690-1697, 1999.

[27] J. Joss and A. Waldvogel, "A raindrop spectrograph with automatic analysis," Pure Appl. Geophys., vol. 68, pp. 240-246, 1967.

[28] J. Hubert, V. N. Bringi, L. D. Carey, and S. Bolen, "CSU-CHILL polarimetric radar measurements in a severe hail storm in eastern Colorado," J. Appl. Meteor., vol. 37, pp. 749-775, 1998.

[29] Z. Hu and R. C. Srivastava, "Evolution of raindrop size distribution by coalescence, breakup and evaporation: Theory and observations," $J$. Atmos. Sci., vol. 52, pp. 1781-1783, 1995.

[30] T. A. Seliga, K. Aydin, and H. Direskeneli, "Disdrometer measurements during an intense rainfall event in central Illinois: Implications for differential reflectivity radar observations," J. Clim. Appl. Meteor., vol. 25 , pp. 835-846, 1986.

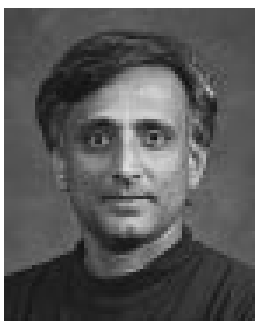

V. N. Bringi received the B.Tech. (hons.) from the Indian Institute of Technology, Mumbai, India, and the $\mathrm{Ph} . \mathrm{D}$. in electrical engineering from The Ohio State University, Columbus, in 1976.

$\mathrm{He}$ is currently a Professor of electrical and computer engineering with Colorado State University, Fort Collins. He is a pioneer in the field of polarimetric radar applied to meteorology and has published over 60 journal articles. More recently, he has written the book Polarimetric Doppler Weather Radar: Principles and Applications with Prof. V. Chandrasekar (Cambridge Univ. Press: New York).

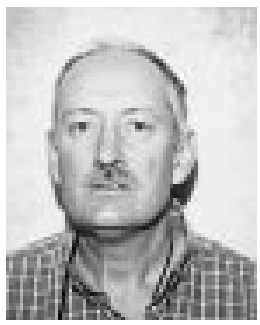

T. D. Keenan received the B.Sc. (hons.) degree in physics and the Ph.D. degree, both from the University of Melbourne, Melbourne, Australia, in 1971 and 1978 , respectively.

He is currently a Senior Principal Research Scientist with the Bureau of Meteorology Research Center, Australia, where he is Leader of the Weather Forecast Group. He is undertaking research on radar meteorology, validation of satellite rainfall measurements, aviation weather, and nowcasting.

Dr. Keenan is a member of the American Meteorological Society, the Royal Meteorological Society, and the Australian Atmosphere and Oceanographic Society.

V. Chandrasekar (S'83-M'87) is currently a Professor with the Department of Electrical Engineering, Colorado State University, Fort Collins. 